\title{
Multiple sensitivity phenotype in interstitial cystitis/bladder pain syndrome
}

\author{
Michael B. Fuoco, MD; Karen Irvine-Bird, MD; J. Curtis Nickel, MD, FRCSC
}

Department of Urology, Queen's University, Kingston, ON

Cite as: Can Urol Assoc J 2014;8(11-12):e758-61. http://dx.doi.org/10.5489/cuaj.2031

Published online November 24, 2014.

\section{Abstract}

Introduction: Phenotypic differentiation of patients with interstitial cystitis/bladder pain syndrome (IC/BPS) may improve our understanding of the condition, as well as the development of patientspecific treatment strategies. We identified a distinct subgroup of IC/BPS patients with a multiple sensitivity phenotype.

Methods: We defined patients with this IC/BPS associated multiple sensitivity syndrome as having at least 3 confirmed allergies/sensitivities to medications and/or environmental factors and a diagnosis of IC/BPS. These IC/BPS patients identified with a multiple sensitivity phenotype (cases) were compared to agematched IC/BPS patients with few or no allergies (controls) at a $1: 2$ ratio. Comparisons were undertaken using standardized case assessment parameters (age, duration of symptoms, medical history, Interstitial Cystitis Symptoms Index [ICSI] and pelvic pain and urinary urgency/frequency [PUF] symptom scores, and urinary, psychosocial, organ specific, infection, neurologic/systemic, tenderness [UPOINT] categorization).

Results: The study consisted of 17 cases and 34 age-matched controls; the mean age was 55 and 56 years, respectively. There was statistically more medication and environmental allergies in the cases versus controls. Cases reported more concomitant illnesses (9.6 vs. 6.2, $p<0.001)$ and number of bodily systems affected (6.0 vs. $3.8, p \leq 0.001)$. The prevalence of irritable bowel syndrome and fibromyalgia was higher in the case group $(p=0.028, p \leq 0.001$, respectively). Additionally, there were more reported psychiatric diseases $(p=0.019)$, allergic/immune diseases $(p=0.003)$, and pulmonary diseases $(p<0.001)$ in the case group. UPOINT classification differed with more patients in the case group being categorized in the psychosocial and neuropathic/systemic domains $(p=0.045, p=0.007$, respectively). Total UPOINT classification (out of 6 ) was also higher in cases than controls (4.6 vs. 3.2, $p=0.001$, respectively).

Conclusions: We have characterized a distinct phenotypic group of patients with IC/BPS and multiple sensitivities. The limitations of our study include the retrospective case-control matching design, biases in phenotype definition, single centre patient recruitment, and the lack of follow-up. Nonetheless, the observation of this specific phenotype suggests that further research in this group may help develop targeted therapeutic strategies for patients with a concomitant multiple sensitivity syndrome and IC/BPS.

\section{Introduction}

Interstitial cystitis/bladder pain syndrome (IC/BPS) is a chronic pelvic pain condition with pain perceived to be due to bladder and storage urinary symptoms. Many patients also have other associated regional and systemic pain syndromes, including irritable bowel syndrome (IBS), fibromyalgia, chronic fatigue syndrome, vulvodynia, headaches, and temperomandibular disorder., ${ }^{1,2}$ For IC/BPS and in particular those with these added conditions, it has been difficult to find consistent and effective treatment despite the significant impact of these conditions on quality of life. The National Institutes of Health/National Institute of Diabetes and Digestive and Kidney Diseases have emphasized the importance of identifying and investigating clinical conditions that overlap with urological chronic pelvic pain syndromes. This emphasis has led to the development of the Multidisciplinary Approach to Pelvic Pain research program. ${ }^{3}$

One approach to examining these overlapping conditions is clinical phenotyping. By developing groupings of patients into specifically defined categories of urologic pelvic pain patients, one would hope to have more targeted and effective therapies directed towards the unique disease profiles exhibited by each phenotypic grouping. The UPOINT (Urinary, Psychosocial, Organ specific, Infection, Neurologic/Systemic, Tenderness) is an example of a clinical phenotyping classification system that has been clinically validated in interstitial cystitis. ${ }^{4}$ It is worth noting that phenotypes expressed outside the bladder (non-urologic) have the most significant impact on quality of life.

Our database of patients with IC/BPS included patients who seemed to have an inordinate number of allergies and 
sensitivities. There is some evidence suggesting a connection between allergic disorders and IC/BPS. Mast cells have been frequently reported to be associated with IC/BPS. ${ }^{5}$ Yamada has reported that as many as $86 \%$ of 36 young study patients with IC/BPS had allergic complications. ${ }^{6}$ There are also case reports of management of a patient's allergic disease concomitantly leading to relief of their IC/BPS symptoms. ${ }^{7,8} \mathrm{We}$ believed, based on our ongoing clinical impression, that another relevant clinical phenotype would include these patients with multiple sensitivity syndromes.

We investigated our select group of patients to see if they fit a new clinical phenotype in IC/BPS. We used a casecontrol design study to examine the phenotype of patients with IC/BPS and multiple allergies as compared to patients with IC/BPS and few or no allergies.

\section{Methods}

Cases were identified prospectively in patients assessed at the IC/BPS clinic in Kingston, Ontario. Patients referred to the IC/ BPS clinic completed questionnaires about their medical history, course of disease, symptom scores (Interstitial Cystitis Symptoms Index [ICSI] and pelvic pain and urinary urgency/ frequency [PUF]), medications, and allergies. Patients with a confirmed IC/BPS diagnosis, as well as multiple allergies or sensitivites to medications and/or environmental factors, were identified. We defined this IC/BPS associated multiple sensitivity syndrome as having at least 3 confirmed allergies/ sensitivities to medications and/or environmental factors. Age-matched IC/BPS controls with few or no allergies were retrospectively selected from the clinic database. Case to control ratio was $1: 2$.

Collected data included patient age, duration of symptoms, medical history, ICSI and PUF symptom scores, and allergies/sensitivities. Cases and control patients were categorized using the UPOINT clinical phenotyping classification system. Medical history was categorized by the affected organ system. Specific syndromes commonly associated with IC/BPS, such as IBS, fibromyalgia, chronic fatigue and depression, were documented separately.

A two-tailed T-test was used to compare age, duration of symptoms, number of allergies, and number of reported illnesses. A Fisher's exact test was employed to compare specific diseases, each organ system affected, and individual UPOINT scores for each domain. For symptom scores and total UPOINT score, the Mann Whitney U test was used.

\section{Results}

In total, 17 cases were compared to 34 age-matched controls (Table 1). Case and control patient ages (mean 55.9 and 54.9 , respectively), and duration of symptoms (mean 5.5 and 9.0 years, respectively) were not significantly different
( $p=0.81$ and $p=0.15$, respectively). As expected by definition of case and control categories, there was a significant difference between groups with respect to number of allergies/sensitivities. This was true for all categories of allergies/ sensitivities, including antibiotics, other medications (not antibiotics), all medications, environmental/food allergies, and all allergies ( $p<0.01$ for all).

There was a significant difference between cases and controls comparing the number of non-bladder systems affected (mean 6.00 vs. 3.82, $p<0.01$ ). There was also a significant difference between number of reported illnesses (mean 9.59 vs. 6.18, $p<0.01)$. Specifically, in the case group there was significantly more IBS (59\% vs. $24 \%, p=0.03$ ) and fibromyalgia $(59 \%$ vs. $9 \%, p<0.01)$ than in the control group of IC/BPS patients. There was no significant difference between groups in the prevalence of chronic fatigue or depression. When considering organ systems, the cases had significantly more psychiatric (47\% vs. $15 \%, p=0.02)$, allergic/immune (47\% vs. $9 \%, p<0.01)$, and pulmonary disease $(88 \%$ vs $12 \%, p<0.01$ ) when compared to control patients. Table 2 summarizes the medical history of the two groups of IC/ BPS patients.

There was little difference between cases and controls with respect to symptom scores (Table 3 ). Cases were classified into more total UPOINT domains than controls (mean 4.57 vs. 3.21, $p<0.01$ ). This difference between cases and controls was primarily driven by an increase in the prevalence of the psychosocial (47\% vs. 18\%, $p=0.04$ ) and neurologic/systemic (71\% vs. 29\%, $p=0.01$ ) domains.

\section{Discussion}

Identified IC/BPS cases with multiple allergies and sensitivities appear to be a distinct clinical phenotype compared to age-matched IC/BPS control patients. Patients with multiple sensitivities and IC/BPS had significantly more organ systems affected, specifically pulmonary, allergic/immune,

\begin{tabular}{lccc}
\hline Table 1. Patient characteristics & & \\
\hline & Cases $(\mathbf{n}=\mathbf{1 7})$ & Controls $(\mathbf{n}=\mathbf{3 4})$ & $\boldsymbol{p}$ value \\
\cline { 2 - 4 } & Mean $(95 \% \mathrm{CI})$ & Mean $(95 \% \mathrm{Cl})$ & T-test \\
\hline Age & $\begin{array}{c}55.88(49.79- \\
61.98)\end{array}$ & $\begin{array}{c}54.88(50.06- \\
59.71)\end{array}$ & 0.8093 \\
$\begin{array}{l}\text { Years with } \\
\text { problem }\end{array}$ & $5.53(2.11-8.95)$ & $8.97(5.91-12.03)$ & 0.1496 \\
\hline Allergies & Mean $(95 \% \mathrm{Cl})$ & Mean $(95 \% \mathrm{Cl})$ & T-test \\
\hline Antibiotics & $3.00(2.27-3.73)$ & $0.21(0.07-0.34)$ & $<0.0001$ \\
$\begin{array}{l}\text { Other medications } \\
\text { All medications }\end{array}$ & $4.00(2.00-6.00)$ & $0.06(-0.02-0.14)$ & 0.0014 \\
Environmental/ & $7.00(4.80-9.20)$ & $0.24(0.07-0.40)$ & $<0.0001$ \\
goods & $2.00(1.09-2.91)$ & $0.44(0.22-0.06)$ & 0.0042 \\
All allergies & $9.00(6.93-11.07)$ & $0.68(0.41-0.95)$ & $<0.0001$ \\
\hline
\end{tabular}

$\mathrm{Cl}$ : confidence interval. 
Fuoco et al.

Table 2. Comparing medical history between groups

\begin{tabular}{|c|c|c|c|}
\hline & \multirow{2}{*}{$\begin{array}{l}\text { Cases }(n=17) \\
\text { Mean }(95 \% \mathrm{Cl})\end{array}$} & \multirow{2}{*}{$\begin{array}{c}\text { Controls }(\mathbf{n}=\mathbf{3 4}) \\
\text { Mean }(95 \% \mathrm{Cl})\end{array}$} & \multirow{2}{*}{$\begin{array}{c}p \text { value } \\
\text { T-test }\end{array}$} \\
\hline & & & \\
\hline $\begin{array}{l}\text { Number of systems } \\
\text { affected (/12) }\end{array}$ & $6.00(5.35-6.65)$ & $3.82(3.34-4.30)$ & $<0.0001$ \\
\hline \multirow[t]{2}{*}{$\begin{array}{l}\text { Number of } \\
\text { reported illnesses }\end{array}$} & $\begin{array}{l}9.59(8.39- \\
10.79)\end{array}$ & $6.18(5.35-7.00)$ & $<0.0001$ \\
\hline & With disease & With disease & $\begin{array}{c}\text { Fisher's } \\
\text { exact }\end{array}$ \\
\hline IBS & $59 \%$ & $24 \%$ & 0.0276 \\
\hline Fibromyalgia & $59 \%$ & $9 \%$ & 0.0003 \\
\hline Chronic fatigue & $18 \%$ & $9 \%$ & 0.3871 \\
\hline Depression & $35 \%$ & $15 \%$ & 0.1474 \\
\hline GU disease & $71 \%$ & $85 \%$ & 0.2700 \\
\hline Neurologic disease & $35 \%$ & $44 \%$ & 0.7635 \\
\hline $\begin{array}{l}\text { Hematologic } \\
\text { disease }\end{array}$ & $18 \%$ & $6 \%$ & 0.3179 \\
\hline Psychiatric disease & $47 \%$ & $15 \%$ & 0.0188 \\
\hline GI disease & $88 \%$ & $71 \%$ & 0.2934 \\
\hline ENT disease & $41 \%$ & $15 \%$ & 0.0763 \\
\hline $\begin{array}{l}\text { Allergic/immune } \\
\text { disease }\end{array}$ & $47 \%$ & $9 \%$ & 0.0034 \\
\hline MSK disease & $82 \%$ & $59 \%$ & 0.1221 \\
\hline $\begin{array}{l}\text { Dermatologic } \\
\text { disease }\end{array}$ & $6 \%$ & $6 \%$ & 1.0000 \\
\hline $\begin{array}{l}\text { Cardiovascular } \\
\text { disease }\end{array}$ & $29 \%$ & $38 \%$ & 0.7568 \\
\hline Pulmonary disease & $88 \%$ & $12 \%$ & $<0.0001$ \\
\hline $\begin{array}{l}\text { Endocrinologic } \\
\text { disease }\end{array}$ & $47 \%$ & $24 \%$ & 0.1150 \\
\hline
\end{tabular}

and psychiatric disease. A hypersensitivity phenotype would be expected to be diagnosed with more allergic/immune disease (by inclusion definition) and the pulmonary correlation is likely secondary to the prevalence of reactive air- way disease seen in this category of patients. An association with psychiatric disease has been described for IC/BPS in the past, though the patients with multiple sensitivities had significantly more psychiatric disease than the controls. It could be speculated that increased psychological stressors may be due to the increased burden of illness and disability.

Cases had an increased prevalence of being categorized into the psychosocial and neurologic/systemic UPOINT domains. This correlates with the increased risk of nonbladder conditions, including an increased prevalence of IBS and fibromyalgia. However, disease specific symptom scores (related to the bladder centric syndrome) are not different between cases with multiple sensitivities and those control patients without multiple sensitivities. The data would suggest that our case phenotype has similar IC/BPS symptomatology, but also further associated systemic conditions. Further etiology studies will be needed to substantiate an etiological difference between these IC/BPS patient groups. Eventually a biomarker may be found to validate this clinical and likely etiological differentiation.

The clinical relevance of this study is based on improved diagnostic categorization and hopefully improved management of specific IC/BPS populations. Patients being evaluated for a diagnosis of IC/BPS should be assessed for multiple allergies/sensitivities. Those identified with a multisensitivity phenotype should be carefully assessed for psychological, bowel, generalized pain, and pulmonary symptoms. These factors could help identify specific non-bladder and systemic conditions we have observed in this study, specifically psychological coping, irritable bowel disease, fibromyalgia, and pulmonary disease. By treating only the bladder symptoms of patients with this multiple sensitivity phenotype, we will likely fail to help the patient. These other conditions need to be addressed by the urologist, the family physician and in many cases by specific specialists working collabora-

Table 3. Comparing symptom score and UPOINT classification between groups

\begin{tabular}{|c|c|c|c|}
\hline & Cases $(n=17)$ & Controls $(n=34)$ & $p$ value \\
\hline Symptoms & Mean (95\% Cl) & Mean (95\% Cl) & Mann-Whitney U \\
\hline ICSI Symptom Score (/19) & $14.29(12.30-16.27)$ & $12.63(11.33-13.92)$ & 0.2932 \\
\hline ICSI Problem Score (/16) & $12.21(10.63-13.80)$ & $10.69(9.56-11.82)$ & 0.1775 \\
\hline PUF Score (/35) & $21.36(19.44-23.28)$ & $17.88(15.94-19.81)$ & 0.4714 \\
\hline UPOINT & With domain affected & With domain affected & Fisher's exact \\
\hline Urinary (U) & $100 \%$ & $100 \%$ & 1.0000 \\
\hline Psychosocial (P) & $47 \%$ & $18 \%$ & 0.0446 \\
\hline Organ Specific (O) & $100 \%$ & $100 \%$ & 1.0000 \\
\hline Infection (I) & $47 \%$ & $24 \%$ & 0.1150 \\
\hline Neurologic/Systemic (N) & $71 \%$ & $29 \%$ & 0.0074 \\
\hline \multirow[t]{2}{*}{ Tenderness $(T)$} & $65 \%$ & $38 \%$ & 0.1357 \\
\hline & Mean $(95 \% \mathrm{Cl})$ & Mean $(95 \% \mathrm{Cl})$ & Mann-Whitney U \\
\hline Total (/6) & $4.57(3.95-5.19)$ & $3.21(2.94-3.49)$ & 0.0009 \\
\hline
\end{tabular}

UPOINT: Urinary, Psychosocial, Organ specific, Infection, Neurologic/Systemic, Tenderness; ICSI: Interstitial Cystitis Symptoms Index; PUF: Pelvic pain and urinary urgency/frequency; Cl: confidence interval. 
tively to treat the "whole" patient. It is also possible that therapy for one condition will decrease the impact of the associated syndrome symptoms; for example, reduction of bladder pain may result in less psychological distress and treatment of allergic rhinitis can improve urinary symptoms in patients with IC/BPS, ${ }^{7}$ particularly if the etiologic pathways are related.

The very real limitations of our study include the retrospective case-control matching design, biases in phenotype definition, single centre patient recruitment, and the lack of follow-up, specifically with respect to phenotype directed therapy. However, our observations mirror those seen in many clinical series described by many urologists who manage IC/BPS. If validated by examination of larger databases of phenotyped patients (for example the NIH MAP Epidemiology-Phenotyping Study), our study may allow us to better understand this complicated and heterogenous condition and to eventually improve therapy.

\section{Conclusion}

We have characterized a distinct phenotypic group of patients with IC/BPS and multiple sensitivities. Besides allergic disease, these patients have more associated nonbladder syndromes and conditions. The observation and clinical differentiation of this specific phenotype suggests further research directions in the etiology and pathogenesis of this group and may help in developing phenotype targeted therapeutic strategies for a patients with a concomitant multiple sensitivity syndrome and IC/BPS.
Competing interests: Dr. Fuoco and Dr. Irvine-Bird declare no competing financial or personal interests. Dr. Nickel is a consultant for Glaxo-Smith-Kline, Taris Biomedical, Pfizer, Eli Lilly, Johnson\&Johnson, Farr Labs, Astellas, Trillium Therapeutics, Auxillium, Ferring and Tribute. He has also participated in clinical trials with Glaxo-Smith-Kline, Taris Biomedical, Pfizer and Eli Lilly.

This paper has been peer-reviewed.

\section{References}

1. Nickel JC, Tripp DA, Pontari M, et al. Interstitial cystitis/painful bladder syndrome and associated medical conditions with an emphasis on irritable bowel syndrome, fibromyalgia and chronic fatigue syndrome. J Urol 2010;184:1358-63. http://dx.doi.org/10.1016/i.juro.2010.06.005

2. Rodriguez MA, Afari N, Buchwald DS. Evidence for overlap between urological and nonurological unexplained clinical conditions. J Urol 2009;182:2123. http://dx.doi.org/10.1016/i.juro.2009.07.036

3. Multi-Disciplinary Approach to the Study of Chronic Pelvic Pain Web site. www.mappnetwork.org. Accessed October 29, 2014.

4. Nickel JC, Shoskes D, Irvine-Bird K. Clinical phenotyping of women with interstitial cystitis/painful bladder syndrome: A key to classification and potentially improved management. J Urol 2009;182:155-60. http://dx.doi.org/10.1016/i.juro.2009.02.122

5. Hanno PM. Bladder pain syndrome (intersititial cystitis) and related disorders. In: Kavoussi LR, Novick AC, Partin AW, Peters CA, eds. Campbell Walsh Urology. 10th ed. Philadelphia, PA: Elsevier Saunders; 2012:371. http://dx.doi.org/10.1016/B978-1-4160-6911-9.00012-8

6. Yamada T. Significance of complications of allergic diseases in young patients with interstitial cystitis. Int J Urol 2003;10:S56-8. http://dx.doi.10.1046/i.1442-2042.10.s1.12.x

7. Traut IL, Macdonald ES, Spangler ML, et al. Montelukast for symptom control of interstitial cystitis. Ann Pharmacother 2011;45:e49. hitp://dx.doi.org/10.1345/aph.1Q130

8. Lee J, Doggweiler-Wiygul R, Kim S, et al. Is interstitial cystitis an allergic disorder?: A case of interstitial cystitis treated successfully with anti-lgE. Int J Urol 2006;13:631-4. http://dx.doi.org/10.1111/j.14422042.2006.01373.x

Correspondence: Dr. Michael Fuoco, Department of Urology, Queen's University, Kingston, ON; mfuoco@qmed.co 